\title{
Morfologia de esporos de pteridófitas do Parque Estadual das Fontes do Ipiranga (São Paulo, Brasil)
}

\author{
Família: 5-Dennstaedtiaceae
}

\author{
Luciano Mauricio Esteves ${ }^{1,2}$ e Carolina Brandão Coelho ${ }^{1}$
}

Recebido: 4.09.2006; aceito: 06.06.2007

\begin{abstract}
Morphology of spores of pteridophyte from the "Parque Estadual das Fontes do Ipiranga" (São Paulo, Brazil): 5. Dennstaedtiaceae). Spores of eleven pteridophyte species of the family Dennstaedtiaceae from the "Parque Estadual das Fontes do Ipiranga" were studied. The family is represented in the area by five genera: Histiopteris, Hypolepis, Lindsaea, Pteridium and Saccoloma. Descriptions, illustrations, observations, as well as an identification key are presented. Key words: Dennstaedtiaceae, morfology, Pteridophyta, spores
\end{abstract}

RESUMO - (Morfologia de esporos de pteridófitas do Parque Estadual das Fontes do Ipiranga (São Paulo, Brasil): 5. Dennstaedtiaceae). Foram estudados os esporos de onze espécies de pteridófitas pertencentes à família Dennstaedtiaceae do Parque Estadual das Fontes do Ipiranga. A família está representada na área por cinco gêneros: Histiopteris, Hypolepis, Lindsaea, Pteridium e Saccoloma. Para todos os táxons estudados são apresentadas descrições, ilustrações, observações, bem como uma chave para a identificação das espécies.

Palavras-chave: Dennstaedtiaceae, esporos, morfologia, Pteridophyta

\section{Introdução}

O presente trabalho faz parte do projeto que estuda a morfologia dos esporos da flora pteridofítica do Parque Estadual das Fontes do Ipiranga (PEFI) e tem como foco a família Dennstaedtiaceae.

A família Dennstaedtiaceae abrange dezoito gêneros (Moran 1995). São plantas terrestres, às vezes epífitas ou hemiepífitas, amplamente distribuídas, ocorrendo em regiões de florestas e habitats alterados (Prado 2004a). A maior parte dos gêneros apresenta esporos triletes. Seis gêneros são monoletes e em Lindsaea é possível encontrar ambos, triletes e monoletes, em um mesmo espécime (Tryon \& Lugardon 1990). Cinco gêneros estão representados na área do Parque Estadual das Fontes do Ipiranga (PEFI), de acordo com levantamento realizado por Prado (2004a): Histiopteris (J. Agardh) J. Sm., Hypolepis Bernh., Lindsaea Dryand. ex Sm., Pteridium Gled. ex Scop., Saccoloma Kaulf. Pretende-se, com o conhecimento de morfologia dos esporos da família Dennstaedtiaceae, complementar os estudos morfológicos e taxonômicos destas espécies presentes no PEFI e ampliar o material de referência para pesquisa sobre precipitação esporopolínica e de sedimentos na área.

\section{Material e métodos}

O planejamento do estudo da presente flora polínica foi baseado em Melhem et al. (1984). Os dados referentes ao Parque Estadual das Fontes do Ipiranga (PEFI) encontram-se nos trabalhos de Melhem et al. $(1981,1984)$. A chave para identificação das espécies da família Dennstaedtiaceae no PEFI foi publicada em Prado (2004b).

Os esporos coletados foram obtidos de material herborizado, depositado no Herbário Científico do Estado "Maria Eneyda P. Kauffmann Fidalgo" (SP). Quando os espécimes apresentavam-se inférteis, ou com quantidade insuficiente de esporos, optou-se por

1. Instituto de Botânica, Caixa Postal 3005, 01061-970 São Paulo, SP, Brasil

2. Autor para correspondência: luciano.esteves@uol.com.br 
coletas a partir de exsicatas provenientes de outras localidades.

Os esporos foram preparados segundo a técnica de acetólise (Erdtman 1960). As ilustrações foram obtidas em microscopia óptica com de esporos acetolisados e fotografados digitalmente, utilizandose fotomicroscópio Olympus BX 50, acoplado a uma câmara de vídeo e microcomputador (PC) e o programa Pro-Plus versão 3 para Windows. Foram medidos os diâmetros: equatorial (E), equatorial maior (EM), equatorial menor (Em), polar (P), o comprimento da lesão (L) e a margem da lesão (ML), determinandose a média, o intervalo de confiança a $95 \%$ e o coeficiente de variabilidade da amostra. A nomenclatura utilizada nas descrições dos esporos é baseada em Lellinger (2002). As lâminas preparadas encontram-se depositadas na Palinoteca da Seção de Dicotiledôneas, Instituto de Botânica.

\section{Resultados e Discussão}

Histiopteris (Agardh) J. Sm.

\section{Histiopteris incisa (Thumb.) J. Sm.}

\section{Figuras 1-3}

Forma: monolete, elipsoidal, côncavo-convexo a plano-convexo em vista equatorial.

Lesão: margem saliente.

Superfície: irregular, com tubérculos parcialmente fundidos e verrugas, exceto na área adjacente à lesão. $\mathrm{O}$ exosporo define o contorno e consiste de duas camadas, a mais externa maior que a interna. Perisporo bastante fino e de difícil visualização sob microscopia óptica.

Medidas ( $\mu \mathrm{m}):$ J. Mattos 14834: $\mathrm{P}=33,6 \pm 0,6$; $\mathrm{EM}=56,0 \pm 0,6 ; \mathrm{Em}=32,2 \pm 0,6 ; \mathrm{L}=33,0 \pm 0,7$.

J. Prado et al. 905: $\mathrm{P}=32,9 \pm 3,5 ; \mathrm{EM}=57,4 \pm$ 2,$9 ; \mathrm{Em}=56,7 \pm 2,1 ; \mathrm{L}=35,8 \pm 2,9$.

Observações: o material $O$. Handro 2193, citado por Prado (2004b), não foi estudado por apresentar esporos em quantidade insuficiente para a análise. Foram estudados os materiais J. Mattos 14834 e J. Prado et al. 905, proveniente de outra localidade.

Material examinado: 27-VII-1967, J. Mattos 14834 (SP); 22-V-1996, J. Prado et al. 905 (SP).

Hypolepis Bernh.

1. Hypolepis repens (L.) C. Presl

Figuras 4-5
Forma: monolete, elipsoidal, plano-convexo em vista equatorial.

Lesão: margem saliente.

Superfície: recoberta por perisporo densamente equinado, com elementos parcialmente fundidos lateralmente, com aspecto semelhante a cristas. Exosporo psilado, com duas camadas de espessuras semelhantes. O perisporo apresenta, além da camada equinada, uma segunda camada interna mais fina.

Medidas $(\mu \mathrm{m}): \mathrm{P}=26,0 \pm 0,7 ; \mathrm{EM}=39,2 \pm 0,6 ;$ $\mathrm{Em}=22,7 \pm 0,8 ; \mathrm{L}=28,1 \pm 0,8$.

Observações: o material $O$. Handro 2185, citado por Prado (2004b), não foi estudado por apresentar esporos em quantidade insuficiente para a análise.

Material examinado: 29-I-1999, M.R. Pietrobom-Silva 4514 (SP).

\section{Lindsaea Dryand. ex Sm.}

Esporos tetraédrico-globosos ou elipsoides; triletes ou raramente monoletes; perisporo psilado, areolado ou rugulado, eventualmente com prolongamentos enovelados; exosporo psilado ou irregularmente tuberculado.

\section{Lindsaea arcuata Kunze}

Figuras 6-8

Forma: trilete, tetraédrico-globoso, com um pequeno número de monoletes e formas intermediárias.

Lesão: margem definida e pouco saliente.

Superfície: exosporo psilado, composto por duas camadas. O perisporo é fino e areolado.

Medidas $(\mu \mathrm{m}): \mathrm{P}=25,9 \pm 0,6 ; \mathrm{E}=31,3 \pm 0,6 ; \mathrm{L}=$ $15,1 \pm 0,6 ; \mathrm{ML}=2,1 \pm 0,1$.

Observações: a presença de monoletes e formas intermediárias indicam uma possível alteração durante o processo da esporogênese. $\mathrm{O}$ material $O$. Handro s.n. (SP50611), citado por Prado (2004b), não foi estudado por apresentar esporos em quantidade insuficiente para a análise.

Material examinado: VI-1965, M. Wacket 1487 (SP).

2. Lindsaea botrychioides A. St.-Hil. Figuras 9-12

Forma: trilete, tetraédrico-globoso.

Lesão: margem definida e saliente.

Superfície: exosporo psilado, composto por duas camadas. $\mathrm{O}$ perisporo é microrrugulado e facilmente 
fraturado ou removido pela acetólise.

Medidas ( $\mu \mathrm{m})$ : O. Handro s.n. (SP46133): $\mathrm{P}=$ $27,0 \pm 0,6 ; \mathrm{E}=29,3 \pm 0,8 ; \mathrm{L}=12,8 \pm 0,6 ; \mathrm{ML}=1,9 \pm$ 0,1 .

G. Davidse \& W.G. Dárcy 10428: $\mathrm{P}=24,9 \pm$ 1,$6 ; \mathrm{E}=27,0 \pm 1,3 ; \mathrm{L}=11,9 \pm 1,8 ; \mathrm{ML}=1,6 \pm 0,2$.

Material examinado: 21-II 1976, G. Davidse \& W.G. Dárcy 10428; 26-VI-1963, O. Handro s.n. (SP46133).

\section{Lindsaea lancea (L.) Bedd. var. lancea}

Figuras 13-16

Forma: trilete, tetraédrico-globoso.

Lesão: margem definida e saliente.

Superfície: exosporo psilado composto por uma camada. Perisporo rugulado e fortemente aderido, com difícil visualização da estratificação.

Medidas $(\mu \mathrm{m}): \mathrm{P}=30,6 \pm 1,1 ; \mathrm{E}=35,5 \pm 1,1 ; \mathrm{L}=$ $15,1 \pm 1,0 ; \mathrm{ML}=3,0 \pm 0,3$.

Observações: os materiais G. Eiten et al.2058, 2079 (SP, US); J.A. Corrêa 13, 58 (SP); J. Prado 1458 (SP), O. Handro 181 (SPF), citados por Prado (2004a), não foram estudados por apresentarem esporos em quantidade insuficiente para a análise.

Material examinado: 7-V-1974, J.A. Corrêa 50 (SP).

4. Lindsaea quadrangularis Raddi ssp. terminalis

K. U. Kramer

Figuras 17-20

Forma: trilete, tetraédrico-globoso, com depressões entre os raios da lesão.

Lesão: margem definida, estreita e saliente.

Superfície: exosporo psilado com uma camada. Perisporo rugulado fortemente aderido, com uma camada

Medidas $(\mu \mathrm{m}): \mathrm{P}=33,1 \pm 0,7 ; \mathrm{E}=39,0 \pm 0,8 ; \mathrm{L}=$ $15,6 \pm 0,6 ; \mathrm{ML}=2,1 \pm 0,2$.

Material examinado: 17-IV-1939, O. Handro s.n. (SP45010).

5. Lindsaea stricta (Sw.) Dryand var. stricta Trans. Linn.

Figuras 21-24

Forma: Trilete, tetraédrico-globoso, com um pequeno número de monoletes e formas intermediárias.

Lesão: margem pouco definida.

Superfície: Exosporo psilado com duas camadas distintas. Perisporo granulado, fino e fortemente aderido ao exosporo.

Medidas $(\mu \mathrm{m}): \mathrm{P}=29,7 \pm 0,8 ; \mathrm{E}=33,9 \pm 1,0 ; \mathrm{L}=$ $14,8 \pm 0,6 ; \mathrm{ML}=5,2 \pm 0,5$.

Observação: a presença de monoletes e formas intermediárias indicam uma possível alteração durante o processo da esporogênese.

Material examinado: 26-VI-1936, F.C. Hoehne s.n. (SP35629).

6. Lindsaea virescens Sw. var. virescens Kungl. Vet. Akad..

Figuras 25-28

Forma: Trilete, tetraédrico-globoso.

Lesão: margem definida e saliente.

Superfície: Exosporo psilado, composto por duas camadas distintas. Perisporo rugulado e fino, fortemente aderido ao exosporo e apenas fraturado pela acetólise.

Medidas ( $\mu \mathrm{m}):$ G. Eiten et al. $2108 A$ (SP, US): $\mathrm{P}=32,9 \pm 2,5 ; \mathrm{E}=35,2 \pm 1,7 ; \mathrm{L}=13,8 \pm 1,7 ; \mathrm{ML}=5,4$ $\pm 1,4$.

J. A. Corrêa 52: $\mathrm{P}=35,3 \pm 2,2 ; \mathrm{E}=38,8 \pm 2,5$; $\mathrm{L}=17,1 \pm 1,3 ; \mathrm{ML}=2,4 \pm 0,1$.

J. A. Corrêa 107: $\mathrm{P}=33,1 \pm 1,5 ; \mathrm{E}=34,0 \pm 1,0$; $\mathrm{L}=15,4 \pm 0,5 ; \mathrm{ML}=2,1 \pm 0,2$.

Observações: o material $O$. Handro s. $n$. (SP35631) (SP), citado por Prado (2004a), não foi estudado por apresentar esporos em quantidade insuficiente para a análise.

Material examinado: 14-VII-1960, G. Eiten et al. 2108A (SP, US); 9-IV-1974, J.A. Corrêa 52 (SP); 9-V-1974, J.A. Corrêa 107 (SP).

\section{Pteridium Scopoli}

1. Pteridium aquilinium (L.) Kuhn var. arachnoideum (Kaulf.) Brade

Figuras 29-32

Forma: trilete, tetraédrico-globoso.

Lesão: sem margem definida.

Superfície: exosporo fino, levemente rugulado. Perisporo granulado e difuso, facilmente removido pela acetólise. $\mathrm{O}$ exosporo fino e o perisporo granulado tornam bastante difíceis as análises da estratificação de ambos.

Medidas ( $\mu \mathrm{m})$ : F.C. Hoehne s. $n$. (SP35694): $\mathrm{P}=$ $32,3 \pm 0,7 ; \mathrm{E}=35,7 \pm 0,7 ; \mathrm{L}=14,5 \pm 0,6 ; \mathrm{ML}=1,6$ $\pm 0,1$.

J. Prado \& D.M. Vital 1429: $\mathrm{P}=30,6 \pm 1,4 ; \mathrm{E}=$ $39,0 \pm 2,3 ; \mathrm{L}=14,4 \pm 1,4 ; \mathrm{ML}=1,5 \pm 0,3$. 
Material examinado: 26-IX-1938, F.C. Hoehne s. $n$. (SP39694); 6-X-2003, J. Prado \& D.M. Vital 1429 (SP).

\section{Saccoloma Kaulf.}

Esporos tetraédricos ou tetraédrico-globosos; trilete; perisporo papilado ou estriado, eventualmente com granulações sobre as estrias; exosporo psilado ou granulado.

\section{Saccoloma elegans Kaulf.}

Figuras 33-36

Forma: trilete, tetraédrico.

Lesão: margem definida e saliente.

Superfície: exosporo granulado com duas camadas. Perisporo com estrias irregulares e próximas, formando sulcos; eventualmente com granulações sobre as estrias; facilmente removido pela acetólise. O perisporo estriado torna bastante difícil a análise do corte ótico e a observação da estratificação.

Medidas: $O$. Handro 2209: $\mathrm{P}=39,2 \pm 1,3 ; \mathrm{E}=42,1 \pm$
0,$9 ; \mathrm{L}=18,5 \pm 0,6 ; \mathrm{ML}=2,4 \pm 0,2$.

M.R. Pietrobom 4238: $\mathrm{P}=38,0 \pm 2,9 ; \mathrm{E}=47,9$ $\pm 2,2 ; \mathrm{L}=20,7 \pm 2,1 ; \mathrm{ML}=3,0 \pm 0,4$.

Material examinado: 25-VII-1972, O. Handro 2209 (SP); 20-IV-1998, M.R. Pietrobom 4238 (SP).

\section{Saccoloma inaequale (Kunze) Mett.} Figuras 37-40

Forma: trilete, tetraédrico-globoso.

Lesão: margem definida e pouco saliente.

Superfície: exosporo granulado. Perisporo com estrias irregulares e sinuosas, facilmente removido pela acetólise. $\mathrm{O}$ exosporo granulado e o perisporo estriado tornam bastante difíceis as análises do corte ótico e a observação da estratificação em ambas.

Medidas: $\mathrm{P}=32,4 \pm 0,8 ; \mathrm{E}=39,5 \pm 0,7 ; \mathrm{L}=15,3$ $\pm 0,6 ; \mathrm{ML}=1,6 \pm 0,1$.

O. Handro 283 (SP, SPF), citado por Prado (2004a), não foi estudado por apresentar esporos em quantidade insuficiente para a análise.

Material examinado: 17-XI-1978, P. Barbour 4519 (SP).

Chave de identificação para as espécies de Dennstaedtiaceae do Parque Estadual das Fontes do Ipiranga

1. Esporos monoletes, elipsoidais

2. Exosporo com tubérculos parcialmente fundidos e verrugas; perisporo fino e difuso .. Histiopteris incisa

2. Exosporo psilado; perisporo densamente equinado Hypolepis repens

1. Esporos triletes

3. Exosporo ornamentado

4. Exosporo granulado

5. Recoberto por estrias irregulares e sinuosas

Saccoloma inaequale

5. Recoberto por estrias irregulares e próximas, formando sulco Saccoloma elegans

4. Exosporo rugulado Pteridium aquilinium

3. Exosporo psilado

6. Perisporo granulado Lindsaea stricta

6. Perisporo sem granulações

7. Perisporo areolado

Lindsaea arcuata

7. Perisporo não areolado

8. Perisporo ornamentado por microrúgulas Lindsaea botrychioides

8. Perisporo ornamentado por rúgulas

9. Depressões entre os raios da lesão Lindsaea quadrangularis

9. Sem depressões entre os raios da lesão

10. Exosporo com uma camada Lindsaea lancea 10. Exosporo com duas camadas Lindsaea virescens 

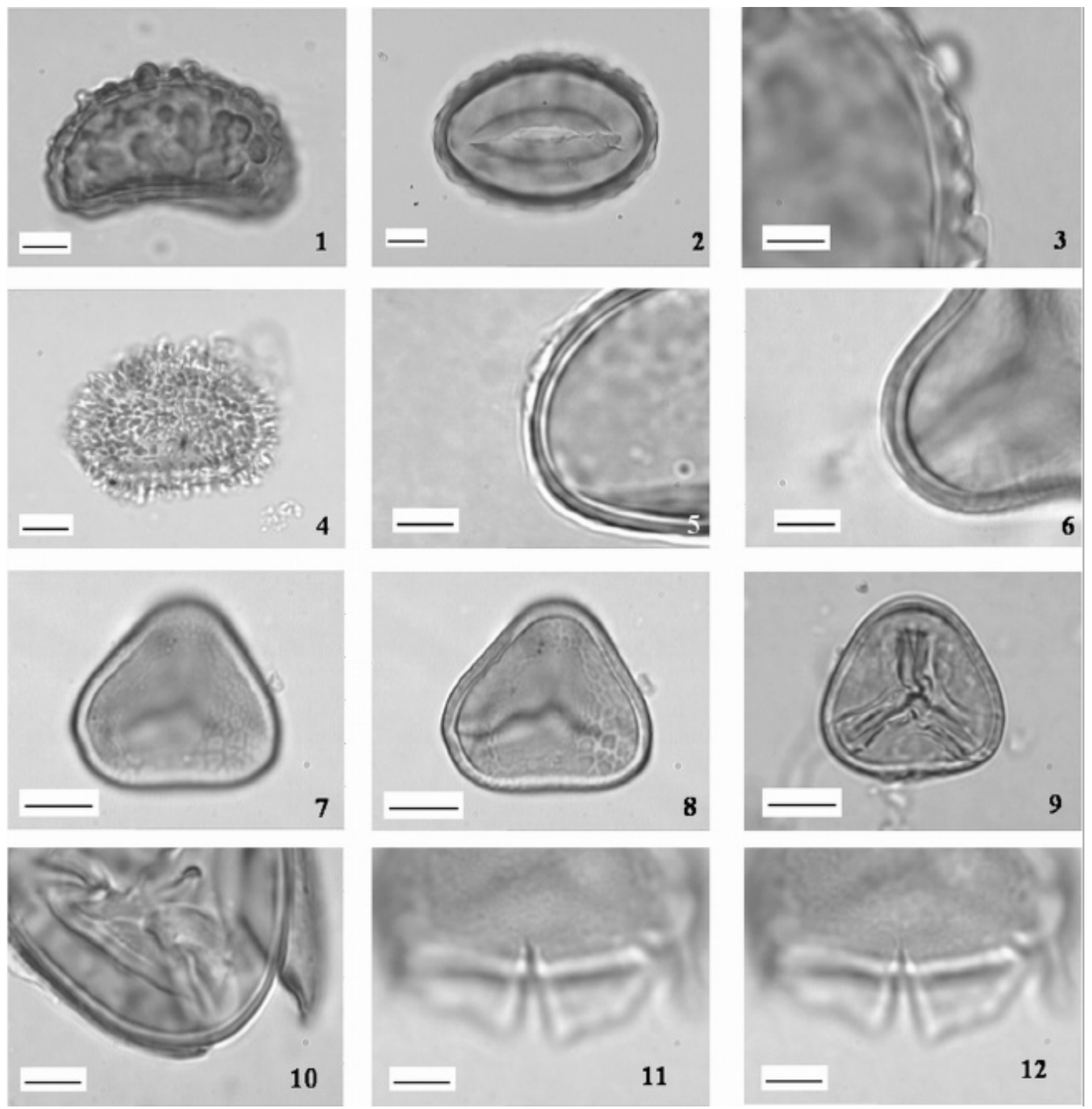

Figuras 1-12. Fotomicrografias de esporos dos gêneros Histiopteris, Hypolepis e Lindsaea (Dennstaedtiaceae). Figuras 1-3. Histiopteris incisa. 1. Vista equatorial, superfície com tubérculos. 2. Vista polar proximal, lesão com margem definida. 3. Corte óptico da parede. Figuras 4-5. Hypolepis repens. 4. Vista equatorial, perisporo equinado. 5. Corte óptico da parede, perisporo parcialmente removido. Figuras 6-8. Lindsaea arcuata. 6. Corte óptico pelo exosporo e perisporo. 7-8. Análise de L.O., foco alto e baixo, respectivamente. Figuras 9-12. Lindsaea botrichioides. 9. Vista polar proximal. 10. Corte óptico da parede com perisporo fraturado. 11-12. Análise de L.O., foco alto e baixo, respectivamente. Escala nas figuras 3, 5-6, 10-12 = 5 $\mu \mathrm{m}$; demais figuras $=10 \mu \mathrm{m}$. 

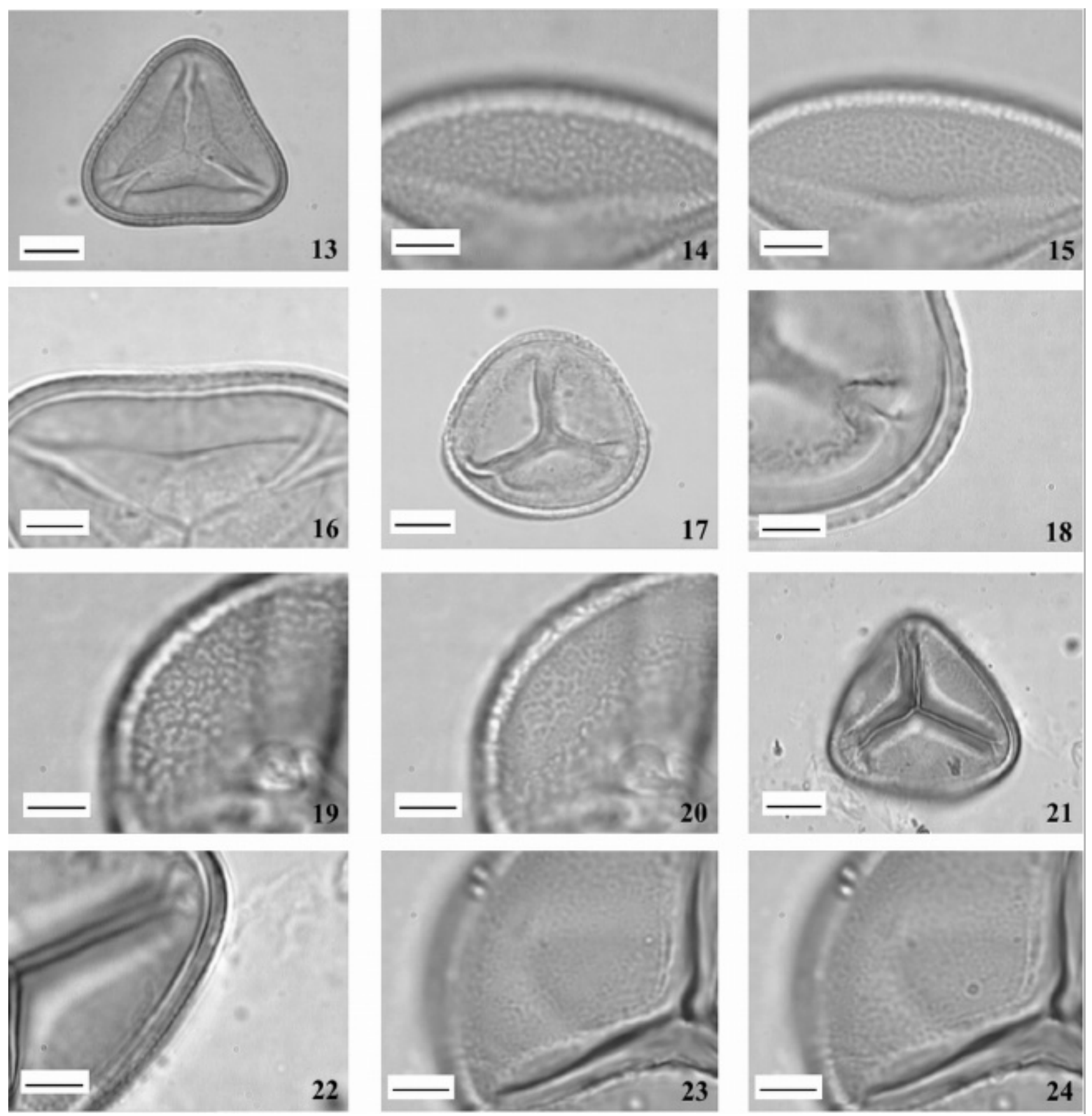

Figuras 13-24. Fotomicrografias de esporos dos gêneros Lindsaea (Dennstaedtiaceae). Figuras 13-16. Lindsaea lancea var. lancea . 13. Vista polar proximal, evidenciando a margem da lesão. 14-15. Análise de L.O., foco alto e baixo, respectivamente. 16. Corte óptico pelo exosporo e perisporo. Figuras 17-20. Lindsaea quadrangularis ssp terminalis. 17. Vista polar proximal, evidenciando depressões entre os raios da lesão. 18. Corte óptico pelo exosporo e perisporo. 19-20. Análise de L.O., foco alto e baixo, respectivamente. Figuras 21-24 Lindsaea stricta var. stricta. 21. Vista polar proximal. 22. Corte óptico pelo exosporo e perisporo. 23-24. Análise de L.O., foco alto e baixo, respectivamente. Escala nas figuras 14-16, 18-20, 22-24 =5 $\mu \mathrm{m}$; demais figuras $=10 \mu \mathrm{m}$. 

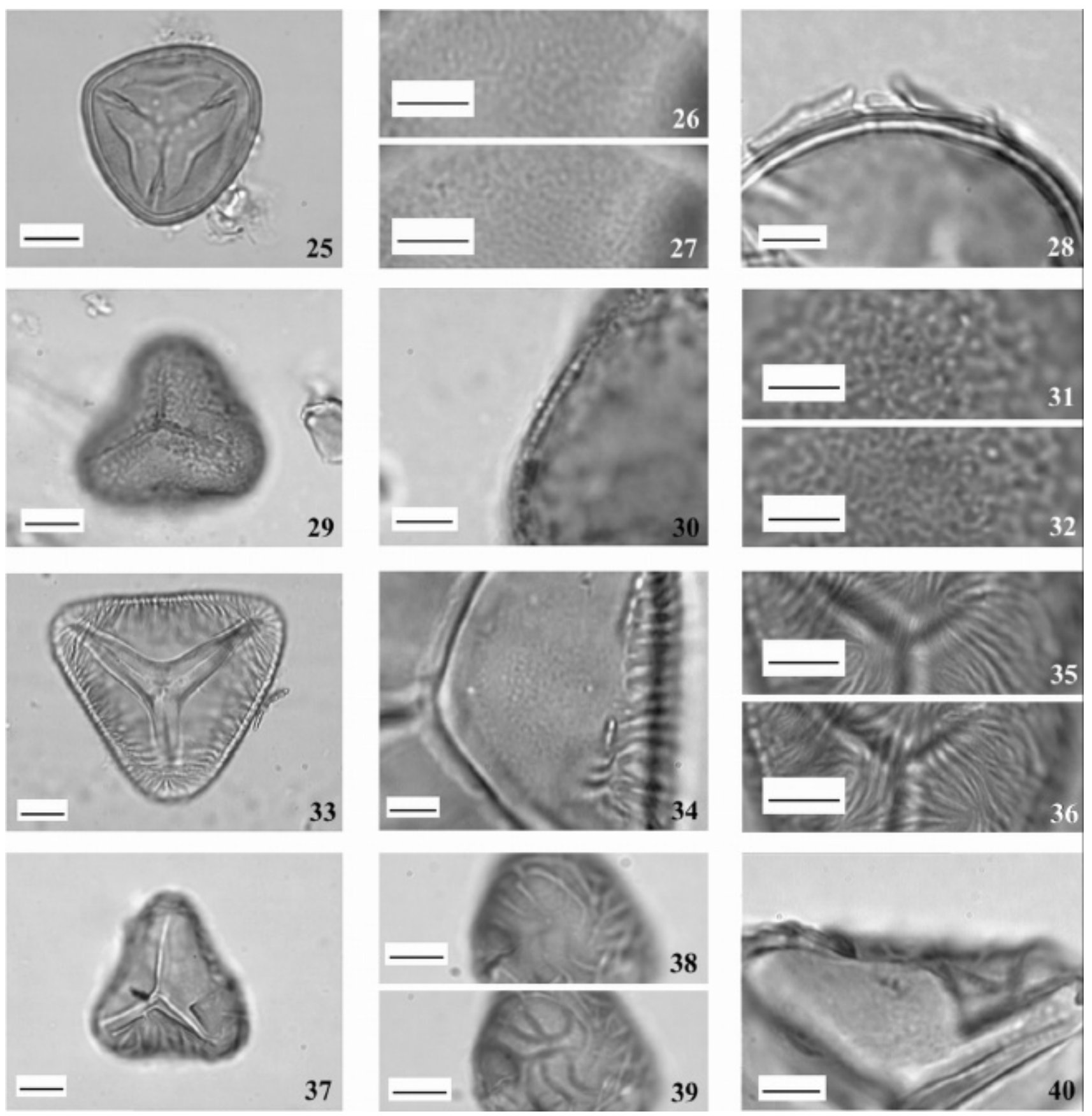

Figuras 25-40. Fotomicrografias de esporos dos gêneros Lindsaea, Pteridium e Saccoloma (Dennstaedtiaceae). Figuras 25-28. Lindsaea virescens var. virescens. 25. Vista polar proximal. 26-27. Análise de L.O., foco alto e baixo, respectivamente. 28. Corte óptico pelo exosporo, evidenciando perisporo fraturado. Figuras 29-32. Pteridium aquilinum var. arachnoideum. 29. Vista polar proximal. 30. Corte óptico da parede. 31-32. Análise de L.O., foco alto e baixo, respectivamente. Figuras 33-36. Saccoloma elegans. 33. Vista polar proximal, contorno e detalhe da lesão. 34. Corte óptico em vista polar proximal, evidenciando exosporo granulado e perisporo estriado fraturado. 35-36 Análise de L.O., foco alto e baixo, respectivamente. Figuras 37-40. Saccoloma inaequale. 37. Vista polar proximal, perisporo fraturado. 38-39. Análise de L.O., foco alto e baixo, respectivamente. 40. Detalhe do exosporo granulado e do perisporo fraturado. Escala nas figuras 26-28, 30-32, 34-36, $40=5 \mu \mathrm{m}$; demais figuras $=10 \mu \mathrm{m}$. 


\section{Agradecimentos}

Os autores agradecem ao Dr. Jefferson Prado pela identificação do material.

\section{Literatura citada}

Erdtman, G. 1960. The acetolysis method. A revised description. Svensk Botanisk Tidskrift 39: 561-564.

Lellinger, D.B. 2002. A modern multilingual glossary for Taxonomic Pteridology. Pteridologia n. 3. Washington, American Fern Society.

Melhem, T.S., Giulietti, M., Forero, E., Barroso, G.M., Silvestre, M.S.F., Jung, S.L., Makino, H., Melo, M.M.R.F., Chiea, S.C., Wanderley, M.G.L., Kirizawa, M. \& Muniz, C. 1981. Planejamento para a elaboração da "Flora Fanerogâmica da Reserva do Parque Estadual das Fontes do Ipiranga (São Paulo, Brasil)". Hoehnea 9: 63-74.
Melhem, T.S., Makino, H., Silvestre, M.S.F., Cruz, M.A.V. \& Jung-Mendaçolli, S. 1984. Planejamento para a elaboração da "Flora Polínica da Reserva do Parque Estadual das Fontes do Ipiranga (São Paulo, Brasil)". Hoehnea 11: 1-7.

Moran, R.C. 1995. Dennstaedtiaceae. In: R.C. Moran \& R. Riba (eds). Psilotaceae e Salviniaceae. In: G. Davidse, M., Sousa, S \& S. Knapp (eds.). Flora Mesoamericana Universidad Nacional Autônoma de México, Ciudad de México, v. 1, pp. 150-163.

Prado, J. 2004a. Criptógamos do Parque Estadual das Fontes do Ipiranga, São Paulo, SP. Pteridophyta: 5. Dennstaedtiaceae. Hoehnea 31: 11-22.

Prado, J. 2004b. Criptógamos do Parque Estadual das Fontes do Ipiranga, São Paulo, SP. Pteridophyta: chave para as famílias: 2. Blechnaceae. Hoehnea 31: 1-10.

Tryon, A.F. \& Lugardon, B. 1990. Spores of the Pteridophyta. Surface, wall structure, and diversity based on electron microscope studies. Springer- Verlag, New York. 